\title{
Exploration of NC Programming Experimental Teaching Based on Reverse Engineering
}

\author{
Xianyi $\mathrm{Li}^{1}$, Xiaoying $\mathrm{Liu}^{2}$ and Senlin Tong ${ }^{1}$ \\ ${ }^{1}$ College of Mechanical and Energy Engineering, Ningbo Institute of Technology, \\ Zhejiang University, Ningbo 315100, China \\ ${ }^{2}$ Zhejiang Fashion Institute of Technology, Ningbo 315211, China \\ Corresponding author e-mail: lxy@nit.zju.edu.cn
}

\begin{abstract}
This paper puts forward the experimental course of the combination of reverse and numerical control programming. In the process of experiment teaching, the LK three coordinate measuring machine(CMM) is used to collect the data of the object, and NX Siemens software is used to realize the CAD inverse modeling, and then the Powermill numerical control programming software is used to carry on the numerical control programming. Through the opening of this experiment, Practice has proved that students not only master the advanced measurement technology, but also master the relevant technology of reverse design and NC programming. It has greatly improved the students' comprehensive application ability and innovation ability, also increased the students' employment competitiveness.
\end{abstract}

\section{Introduction}

Intelligent manufacturing is the main direction of "made-in-china 2025" [1-2], Enterprises urgently need intelligent manufacturing talents. On August 18, 2016, the Ministry announced that Ningbo had become the country's first "made-in-China 2025" pilot demonstration city, at the same time, Ningbo Institute of technology, Zhejiang University will be to upgrade to a high level of application oriented university. So it is necessary to open the experimental course of the combination of reverse engineering and numerical control programming in order to adapt to the development needs of intelligent manufacturing enterprises.

The main experimental equipment used in reverse engineering is the three coordinate measuring machine (CMM). CMM known as the "measurement center"[3-5] is a kind of large scale precision instrument by its versatility, wide measurement range, high precision, good performance, and it can be connected with the flexible manufacturing system. The main equipment of CNC machining is the CNC machine tools, And CNC machine tools are a set of processing equipment which set machine, electricity, liquid, light, computer, automatic control technology as a whole. How to cultivate a high level of numerical control talents with practical ability and corresponding theoretical level is an urgent task for colleges and universities [6].Based on this, the school purchased LK CMMs and CNC milling machines, the corresponding experimental projects are setted up in mechanical and electrical specialty.And a comprehensive, innovative experimental courses are offered through the combination of the two experimental projects. In the course of experiment teaching, firstly, the LK CMM is used to carry out the data collection, Siemens NX software is used to achieve reverse modeling, and then the Powermill numerical control programming software is used to carry on the numerical control programming. Finally, CNC milling machine is used to machine parts. Through the course of the 
experiment, the students' comprehensive application ability and innovation ability are greatly improved, and the students' employment competitiveness is also increased [7-8].

\section{Point Cloud Data Acquisition Based on LK CMM}

Point cloud data acquisition is one of the key technologies in reverse engineering, and it is divided into contact and the non-contact two types. The advantages of contact acquisition are the low cost, less equipment cost, high precision. But the disadvantages are low efficiency, low data amount. It cannot adapt to the complex shape of the object. Non-contact acquisition speed is fast, working distance is far, Non-contact acquisition is not restricted by material, can obtain massive data, can measure complex surface, but the cost is high. In the actual measurement, the appropriate measurement method is chosen according to the actual situation. For example, the contact type measurement is used as far as possible to measure the object whose material is hard and the shape is simple, which is easy to locate. Scanning of the rubber, sludge or thin objects generally use non-contact measurement [9-10].

Data acquisition planning need to be carried out before the point cloud data acquisition, Data acquisition planning refers to the method of determining the data acquisition and the data points, The purpose is the data collected is correct and efficient.

The general steps are as follows: following the characteristic direction, along the normal direction, the important parts are collected accurately, the secondary parts are properly collected, the complex parts are densely collected, the simple parts are sparsely collected. The production profile data is firstly collected, then internal data is gathered [11].

In this experiment, the LK CMM is used to get point cloud data. Specific steps are as Figure 1.

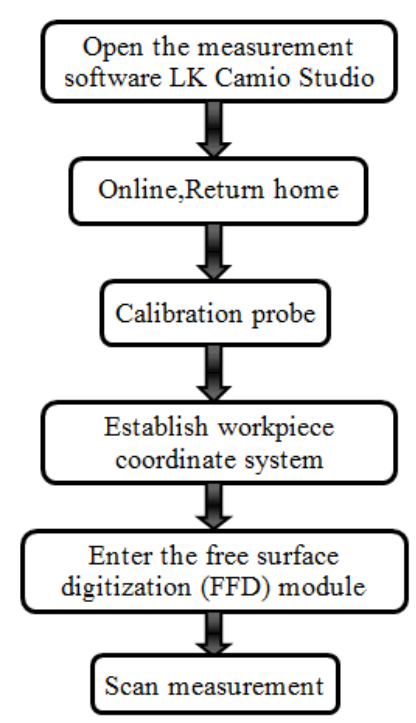

Figure 1. The step of getting point cloud data.

FFD mainly requires the following steps:

Add boundaries to define scanning area.

Determine the direction and spacing of the scanning path by using the grid option to define a grid on the workpiece.

Scan the workpiece to generate point, curve.

Save the data by save as option in the model menu, generally save as .igs format which is easy to be read by the third party software.

The tail box bottom cover of electric bicycle point cloud data which uses the LK CMM to obtain is shown in Figure2.Because the cover is symmetrical, only half of it is scanned. 


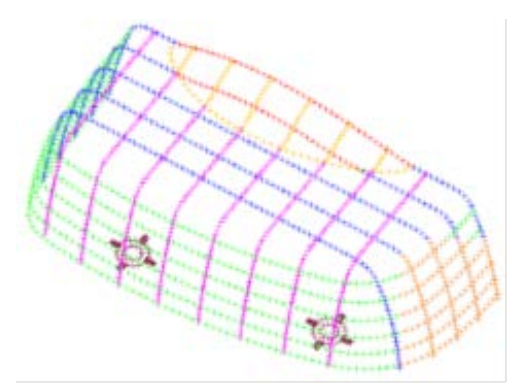

Figure 2. The point cloud data.

\section{Reverse Mmodeling Based on Siemens NX}

There are 3 types of software available for Reverse Engineering. The first is forward engineering software with reverse engineering, such as CREO, NX, CATIA, etc. Second type of choice is the use of special reverse software, such as Imageware, Geomagic Design Direct, CopyCAD, etc. The third is the combination of the special reverse software and the forward software[12].

In this experiment, Siemens NX is used to deal with the data of acquisition point cloud, and the general process is: point processing, line processing, surface treatment, structure processing. NX can read the discrete data points obtained by CMMs, but NX is very difficult to deal with high density point cloud, it need to be discreted into data points in other software and then imported NX.

Before curves are structured through the data, Firstly, points need to be sorted out including noise points, obvious defects. The structured curves are as far as possible to minimize the error and be smooth. Linear circular and spline curve can be used as the connections, spline curve is the most commonly used. Points are be selected as uniform as possible. Rounded corners are firstly ignored and then fillets are rounded. For the measurement error and the matt sample, Curve curvatures often have mutation, so it must be adjusted to smooth.

It is often necessary to adjust the lines or add some curves, so that the curved surface is structured. The surface of the error need to be checked, the surface of the appearance of the higher requirements should be checked the smoothness. The structure of curved surface should pay attention to simplicity. The curved surface should be as large as possible, the number is as small as possible, it cannot be too broken. When the curved surface is built, it is necessary to construct the solid model so as to facilitate the construction of the structure.

The reverse model of the tail box bottom cover is shown in Figure 3.

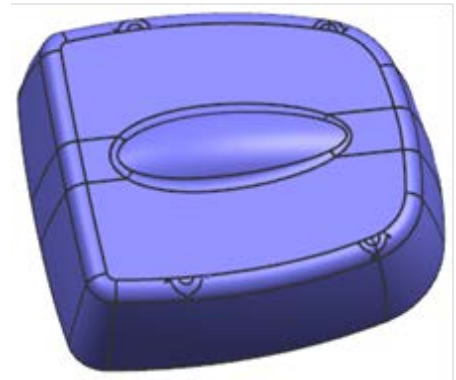

Figure 3. Reverse model of the tail box bottom cover.

\section{NC Programming Based on Powermill}

CNC programming is divided into manual programming and automatic programming. Manual programming mainly complete the various stages of NC programming by manual. Automatic programming is the programming process which uses computers and special software to determine the processing conditions, automatic operation and generating by the way of human-computer interaction. 
Manual programming is mainly for some simple parts programming. The curve profile, 3D surface and other complex parts or dies use automatic programming [13].

Reverse modeling is generally more complex surface, so Delcam's Powermill automatic programming software is used in this experiment. This software is powerful, efficient rough machining, high speed machining and automatic collision avoidance five axis machining strategy. The strategy and functions of the system are in accord with the idea of high speed multi axis machining, which is safe and without overcut, the calculation speed is fast, and the advantages of the direct input of various data formats are supported. It is widely used in parts and dies processing.

The general process of NC programming is as follows:

Accept the processing tasks $\rightarrow$ Inspection and analysis model $\rightarrow$ Clear processing technical requirements $\rightarrow$ Rough machining $\rightarrow$ Second rough machining $\rightarrow$ Local position corner rough machining $\rightarrow$ Processing plane $\rightarrow$ Semi-finishing $\rightarrow$ finishing $\rightarrow$ Corner finishing $\rightarrow$ Machining path edit $\rightarrow$ Cutting parameters inspection $\rightarrow$ overcut inspection $\rightarrow$ Collision inspection $\rightarrow$ simulation processing $\rightarrow$ NC program outputting $\rightarrow$ Generate the programming list.

Through the analysis of the model of the tail box bottom cover, Model area clearance strategy is used to rough machining, Diameter $10 \mathrm{~mm}$ flat cutter is selected. Steep and shallow processing strategy is used to finish the surface of steep and shallow, the tool of diameter $4 \mathrm{~mm}$ is used in the process. Its simulation process is shown in Figure 4.

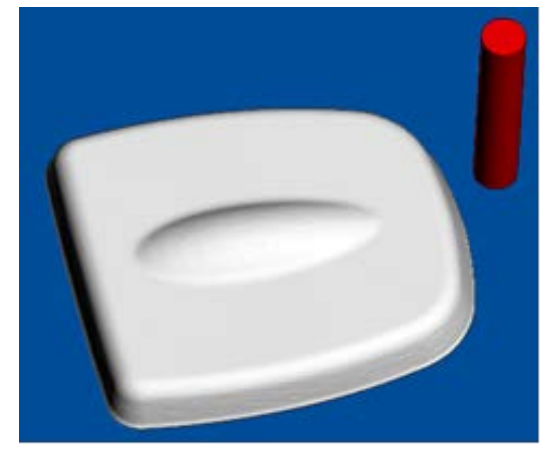

Figure 4. The tail box bottom cover after the simulation processing.

\section{CNC Milling Process}

After the NC program is written, it is necessary to operate the CNC milling machine to process the part.

CNC milling machine general steps are as follows: Starting, Returning to the reference point, Inputting NC program, Editing NC program, Dry running, Tool setting and establishing workpiece coordinate system, Automatic machining, Shutting down.

Tool setting is the key steps in the steps[14-15]. The aim of tool setting is to determine the spatial relationship between the workpiece coordinate system and machine coordinate system through the tool or tool setting tool.

There are a variety of tool setting ways. The tool setting is chosen according to the existing conditions and machining precision. Trial cutting method, side-finding device method, tool setting instrument, tool auto-checking etc. can also be used. The accuracy of trial cutting method is low. Side-finding device and Z tool setting device are commonly used in the machining process. They are efficient and can guarantee the accuracy of tool setting.

The side-finding device is generally used to determine the $\mathrm{X}$ and $\mathrm{Y}$ values of the workpiece coordinate system origin in the machine tool coordinate system. There are eccentric and photoelectric type sidefinding devices, the photoelectric type is more commonly used. The probe of photoelectric is generally $10 \mathrm{~mm}$ steel ball which is pulled taut in the measuring rod of photoelectric side-finding device by a spring. When the probe touches the work piece, it will retreat, and the circuit is switched on and the 
light signal is sent out. The coordinate position of the measured surface can be obtained by the indication of the photoelectric side-finding device and the machine coordinate position.

The $\mathrm{Z}$ axis setting device is commonly used to determine the $\mathrm{Z}$ axis coordinate of the workpiece coordinate system origin in the machine tool coordinate system. The $\mathrm{Z}$ axis setting device is provided with a photoelectric type and a pointer type. It determines whether a cutting tool and the setting device contact through photoelectric instructions or pointer. The precision can reach $0.005 \mathrm{~mm}$ in general. The $\mathrm{Z}$ axis setting device is provided with the magnetic table blocks which can firmly adhere to the workpiece or fixture, its height is commonly $50 \mathrm{~mm}$ or $100 \mathrm{~mm}$.

According to the above steps, the numerical control milling machine is operated. Because the tail box cover is too big, it is processed after a certain proportion of the scale. The processing process is shown in Figure 5.

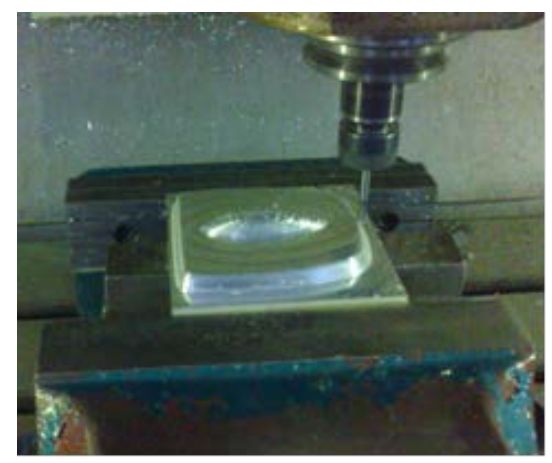

Figure 5. Processing of the tail box bottom cover.

\section{Conclusion}

The experimental course is a comprehensive and innovative experiment on the combination of reverse engineering and numerical control programming. The experiment is not limited to the tail box bottom cover, Students can freely choose, such as mice, toys, etc. This experimental course can realize the teaching mode which is based on the students' self-training, improve students' innovative thinking and practical ability, cultivate students' scientific attitude and pioneering innovation consciousness, fully mobilize students' initiative, enthusiasm and creativity, improve the students' interest, train the students' comprehensive application ability. At the same time, it also improves the students' ability to compete for employment.

\section{Acknowledgments}

This work was financially supported by Ningbo Institute of Technology, Zhejiang University teaching research and reform project (No.NITJG-201633).

\section{References}

[1] Zhou Ji, Intelligent manufacturing main direction of “Made in China 2025”,J.China Mechanical Engineering.26 (2015) 2273-2284.

[2] Fu Jianzhong, Development status and trend of intelligent manufacturing equipment, J.Journal of Mechanical\&Electrical Engineering. 31 (2014) 959-962.

[3] Wang Hongtao, Zhou Yaoxin, High duality experiment teaching with the coordinate measuring machine, J.Laboratory Research and Exploration. 6 (2000) 27-28,32.

[4] Jin Tao, Chen Jianliang, Tong Shuiguang, A review of reverse engineering technology, J. China Mechanical Engineering. 13 (2002) 1430-1436.

[5] Zhang Guoxiong, Coordinate Measuring Machine, Tianjin University Press, Tianjin, 1999.

[6] Gou Xiangfeng, Yang Jin, Chen Dedao,etc, Design and practice of open all-round CNC machining experiment, J. Laboratory Research and Exploration. 30 (2011) 137-139,144. 
[7] Huang Daofeng, Li Huaijian, Zhu Yuhua, Some measures to improve experimental teaching quality,J. Laboratory Research and Exploration. 29 (2010) 120-122.

[8] Ci Ruimei,Zhang Xiang,Zuo Qian, A probe into building the reverse engineering laboratory in higher vocational colleges, J. Journal of Yangzhou Polytechnic College. 12 (2008) 50-52.

[9] Yang Xuerong, Cheng Siyuan, Ma Dengfu, etc., Exploration and practice of experimental teaching based on coordinate measuring machine, J. Laboratory Research and Exploration. 30 (2011) 112-115.

[10] Wu Yongqiang, Proficient in UG NX5+Imageware reverse engineering design, Electronics industry Press, Beijing, 2008.

[11] Chen Junmei,Deng Xuexiong,Zhou Min, etc., Data acquisition technology based on coordinate measuring machine in reverse engineering, J. Journal of Mechanical\&electrical technology. 33 (2004) 82-84.

[12] Hexagon Measurement Technology (Qingdao) Co., Ltd, The Practical Coordinate Measuring Technology, Chemical Industry Press, Beijing, 2007.

[13] Yang Shurong, Zhao Yan, Liang Heng, PowerMILL NC programming tutorial-the basic article, Machinery Industry Press, Beijing, 2013.

[14] Zheng Zhenping,Yu De,Zhang Xiaohong, NX UG5 typical examples of CNC machining, Electronic Industry Press, Beijing, 2007.

[15] Gao Changyin, Zang Wentong, Zhao Wen, UG NX6.0 CNC five axis machining examples tutorial, Chemical Industry Press, Beijing, 2009. 